\section{A organização da Vigilância \\ Alimentar e Nutricional no \\ Sistema Único de Saúde: \\ histórico e desafios atuais}

Organization of Food and

Nutritional Surveillance within the

Brazilian National Health System:

history and current challenges

Janine Giuberti Coutinho'

Antônio José Costa Cardoso"

Natacha Toral"'

Ana Carolina Feldenheimer da Silva"I'

Juliana Amorim Ubarana"I

Kelva Karina Nogueira de Carvalho de Aquino"'

Eduardo Augusto Fernandes Nilson"'

Antônio Fagundes"'I

Ana Beatriz Vasconcellos"'I

'Coordenação-Geral da Política de Alimentação e Nutrição-CGPAN/DAB/SAS/ MS e Observatório de Políticas de Segurança Alimentar e Nutrição/UnB.

"Departamento de Saúde Coletiva da Universidade de Brasília.

"'Coordenação-Geral da Política de Alimentação e Nutrição - CGPAN/

Departamento de Atenção Básica/ Secretaria de Atenção à Saúde/ Ministério da Saúde.

\section{Resumo}

A Política Nacional de Alimentação e Nutrição ressalta a importância do Sistema de Vigilância Alimentar e Nutricional-SISVAN, que se destina à identificação do diagnóstico descritivo e analítico da situação alimentar e nutricional da população brasileira, contribuindo para que se conheça a natureza e a magnitude dos problemas nutricionais do país. Este artigo visa apresentar o histórico das ações da Vigilância Alimentar e Nutricional no Brasil, desde sua idealização até a prática atual, destacando a superação das limitações encontradas ao longo dos anos, o fortalecimento obtido por meio dos marcos legais e os desafios enfrentados neste momento. Entende-se hoje que o SISVAN só pode ser conduzido utilizando-se uma combinação de estratégias, sendo as principais: o sistema informatizado do SISVAN, os inquéritos populacionais, as Chamadas Nutricionais, a análise das bases de dados nacionais da saúde, o acesso à produção científica e o financiamento de pesquisas sobre o tema.

Palavras-chave:Vigilância Nutricional. Monitoramento. Estado nutricional. Consumo alimentar. 


\section{Abstract}

The National Food and Nutrition Policy emphasizes the importance of the Food and Nutritional Surveillance System (SISVAN), which has the purpose of determining descriptive and analytical diagnoses of the food and nutritional status of the Brazilian population. It thus contributes towards providing knowledge on the nature and magnitude of the country's nutritional problems. The present paper aimed to present the history of food and nutritional surveillance actions in Brazil, from the time of concept design to current practice. The surmounting limitations encountered over the years, strengthening obtained through legal milestones and challenges currently faced are highlighted. The view taken is that SISVAN can only be managed today through a combination of strategies. Of these, the main ones are the SISVAN information technology system, population-based surveys, Nutrition Days, and analysis of national healthcare databases, along with access to scientific production and funding for research on the topic.

Keywords: Nutritional Surveillance. Monitoring. Nutritional status. Food consumption.

\section{Introdução}

A Política Nacional de Alimentação e Nutrição (PNAN), aprovada em junho de 1999 pela Portaria no 710, de 10 de junho de 1999, é uma declaração do compromisso do Ministério da Saúde com a erradicação dos males relacionados à falta de alimentos e à pobreza, principalmente a desnutrição infantil e materna, e também o sobrepeso e a obesidade na população adulta ${ }^{1}$. Os propósitos desta política são: assegurar a qualidade dos alimentos disponíveis para o consumo, promover práticas alimentares saudáveis, prevenir e combater os distúrbios nutricionais, e estimular relações intersetoriais a fim de disponibilizar o acesso universal aos alimentos. Uma das diretrizes da PNAN prevê o monitoramento da situação alimentar e nutricional da população que é realizado através do Sistema de Vigilância Alimentar e Nutricional (SISVAN).

AVigilância Alimentar e Nutricional corresponde à descrição contínua e à predição de tendências das condições de alimentação e nutrição da população, assim como de seus fatores determinantes ${ }^{1}$. Abrange atividades de rotina, coletas e análises de dados e informações para descrever as condições alimentares e nutricionais da população. A partir do SISVAN, são embasadas decisões políticas no sentido de auxiliar no planejamento, monitoramento e gerenciamento de programas relacionados com a melhoria dos padrões de consumo alimentar e nutricionais da população.

$\mathrm{O}$ presente material visa apresentar o histórico das ações da Vigilância Alimentar e Nutricional no Brasil, desde sua idealização até a prática atual, destacando a superação das limitações encontradas ao longo dos anos, o fortalecimento obtido por meio dos marcos legais e os desafios enfrentados neste momento.

\section{Vigilância Alimentar e Nutricional: para quê?}

Na lógica do modelo de atenção à saúde atual, a Agenda da Nutrição deve ser orga- 
nizada a partir da caracterização clara do perfil epidemiológico da comunidade e dos espaços domiciliares. Esta ação abrange a identificação de riscos, problemas, prioridades, potencialidades e possibilidades de atuação e reconhecimento da situação de saúde, alimentação e nutrição das famílias. O levantamento epidemiológico da população deve incluir dados provenientes de diversas fontes e sistemas de informação, entre os quais o SISVAN, que servirão de base para a realização do diagnóstico de saúde até a tomada de decisão em diferentes níveis de governo.

A partir do diagnóstico, é possível realizar o planejamento com vistas à estruturação das ações de prevenção e controle das carências nutricionais e de promoção da alimentação saudável no serviço, contemplando as peculiaridades e as diversidades locais. O conhecimento do perfil epidemiológico da população também permite definir os mecanismos de apoio, os espaços intersetoriais e os potenciais atores para uma atuação conjunta da saúde com a educação, a assistência social e o desenvolvimento agrário, entre outros setores.

Além das ações intersetoriais que possibilitam o acesso e a informação sobre alimentação saudável, outras estratégias são adotadas no âmbito da atenção primária à saúde para o cumprimento da Agenda de Nutrição. Estas abrangem a suplementação de micronutrientes para grupos mais vulneráveis como crianças, nutrizes e gestantes, universal ou em regiões endêmicas, cuja carência seja considerada problema de saúde pública (Portarias no 730 , de 13 de maio de 2005, e no 729/GM, de 13 de maio de 2005). Destaca-se também o papel da educação alimentar e nutricional no serviço de saúde, considerando as diferentes fases do curso da vida e as condições de saúde, a partir das diretrizes alimentares oficiais definidas nos guias alimentares.

Para a realização dessas ações em nível local, o Ministério da Saúde adota um modelo de reorientação da atenção primária à saúde, por meio da Estratégia de Saúde da Família, cuja cobertura é crescente em todo o país desde 1994. Trata-se de uma estratégia prioritária que visa atender indivíduos e a família de forma integral e contínua, baseada na territorialização, desenvolvendo ações de promoção, proteção e recuperação da saúde. Tem como objetivo reorganizar a prática assistencial, centrada no hospital, com enfoque na família em seu ambiente físico e social. Também pode ser definido como um modelo de atenção que pressupõe o reconhecimento de saúde como imprescindível na melhoria das condições de vida. Nesse sentido, as equipes de Saúde da Família são consideradas elementos essenciais para apoiar a realização das ações de nutrição no território, incluindo o monitoramento da situação alimentar e nutricional da população atendida.

\section{Histórico da Vigilância Alimentar e Nutricional no Brasil}

A Vigilância Alimentar e Nutricional no Brasil foi preconizada pela primeira vez em 1968, durante a 21 ${ }^{\text {a }}$ Assembléia Mundial de Saúde, atendendo às propostas da Organização Mundial da Saúde - OMS, da Organização Pan-Americana da Saúde OPAS e da Organização das Nações Unidas para a Agricultura e a Alimentação - FAO. Foi sugerido que as ações da Vigilância Epidemiológica não deveriam ser restritas às doenças transmissíveis, mas deveriam ser também aplicáveis a outros problemas de saúde pública, incluindo aqueles relacionados à alimentação e à nutrição.

Em 1974, na Conferência Mundial de Alimentação em Roma, formalizou-se a proposta de vigilância nutricional, fazendo-se uma transposição do conceito de vigilância das enfermidades. Nos países subdesenvolvidos, esta ganhou caráter emergencial, principalmente devido às precárias condições de vida de grupos vulneráveis. $\mathrm{Na}$ ocasião, a Vigilância Nutricional foi apresentada reduzida a um sistema de informação (SI) e de coleta, processamento e análise de informações, com o objetivo de se ter dados contínuos sobre o estado nutricional de populações² . 
Em 1972, mediante a edição da Lei no. 5.829, de 30 de novembro de 1972, foi criado o Instituto Nacional de Alimentação e Nutrição (INAN), uma autarquia do Ministério da Saúde. Quatro anos após a sua criação foi proposta a construção de um Sistema de Informações para a Vigilância Alimentar e Nutricional. Este sistema era direcionado principalmente para o diagnóstico e o tratamento da desnutrição infantil. Contudo, a proposta não foi concretizada em nível nacional, restringindo-se apenas ao desenvolvimento de algumas experiências locais ${ }^{3-5}$.

Em 1975, foi criado o Sistema Nacional de Vigilância Epidemiológica (SNVE) ${ }^{6}$. O contexto político adverso da época contribuiu para que a Vigilância Epidemiológica praticamente se restringisse à notificação compulsória dos casos de doenças transmissíveis, embora a lei conceituasse tal ação de forma mais abrangente.

Nos anos 80 e início dos 90, os processos conjugaram-se no sentido de justificar a Vigilância Epidemiológica da desnutrição, bem como de algumas condições de risco. A promulgação da Lei Orgânica da Saúde (1990), que regulamentou o Sistema Único de Saúde, torna evidente a necessidade de reorganização do SNVE para adequá-lo aos princípios e diretrizes do novo sistema de saúde. É importante ressaltar que essa mesma Lei recomenda a adoção da Vigilância Nutricional no âmbito do SUS, no Capítulo I, artigo 6, inciso IV.

No mesmo ano foi publicada pelo INAN a portaria criando o Sistema de Vigilância Alimentar e Nutricional (Portaria n ${ }^{\circ} .1 .156$, publicada no Diário Oficial da União de 31 de agosto de 1990). E, também no mesmo ano, foi criado o Comitê Assessor para prestar apoio técnico-operacional à sua implementação e um Grupo Técnico de Coordenação para a implementação da Vigilância Alimentar e Nutricional no país²

Contudo, o próprio INAN reconhecia que a Vigilância Alimentar e Nutricional não era executada nos municípios. Assim, deu-se início a uma estreita vinculação do SISVAN aos programas assistenciais do governo, o que marcou a história desta vigilância no país durante muitos anos. Naquele contexto, a realização da vigilância nutricional foi vinculada ao Programa "Leite é Saúde" (Plano de Combate à Fome e à Miséria), com a perspectiva de que esse programa pudesse catalisar a realização das atividades de vigilância nutricional nos municípios. O SISVAN foi definido como requisito para a adesão dos municípios a este Programa, o que restringiu a vigilância nutricional a um instrumento de triagem do Programa².

O INAN foi extinto em 1997, por meio da Medida Provisória no ${ }^{\circ}$. 1.576, de 5 de junho daquele ano ${ }^{3}$, mas, no ano seguinte, o SISVAN foi adotado como um dos prérequisitos para a adesão ao Programa de "Incentivo ao Combate às Carências Nutricionais" - ICCN, de acordo com a Portaria GM/MS 709, publicada no Diário Oficial da União em 11 de junho de 1999. O principal critério de permanência do município no programa era o envio regular de dados às instâncias específicas de vigilância alimentar e nutricional. A cada mês, as Secretarias Municipais de Saúde deveriam enviar os formulários consolidados, preenchidos manualmente, às Secretarias Estaduais de Saúde. Essas, por sua vez, encaminhavam trimestralmente o material para o Ministério da Saúde.

Com a publicação da Portaria no. 710 , de 10 de junho de 1999, foi aprovada a Política Nacional de Alimentação e Nutrição - PNAN ${ }^{1}$. A terceira diretriz determina que a Vigilância Alimentar e Nutricional seja ampliada e aperfeiçoada, de modo a agilizar seus procedimentos e sua cobertura a todo o país, visando dar suporte para o desenho e o ajuste de programas por meio da atualização contínua e avaliação do perfil alimentar e nutricional da população brasileira.

A Política descreve que a Vigilância Alimentar e Nutricional compreenderá a predição contínua de tendências das condições de alimentação e nutrição, bem como de seus fatores determinantes. Aponta também para a compatibilização com outros sistemas de informação do Sistema Único de Saúde, como, por exemplo, o Sistema 
de Informação de Mortalidade (SIM) e o Sistema de Informação de Nascidos Vivos (SINASC). Além disto, sugerem que sejam aproveitados os dias nacionais de vacinação como oportunidades para a realização de estudos sobre alimentação e nutrição.

Para a sua plena realização, de acordo com o previsto na PNAN, no âmbito do Sistema Único de Saúde (SUS), faz-se necessária a implementação da Vigilância Alimentar e Nutricional fomentando a retro-alimentação das ações e políticas. O SISVAN torna-se o principal meio para analisar de forma sistemática a situação alimentar e nutricional do país, de forma a gerar o feedback entre a informação, a ação e a análise dos resultados ${ }^{1}$.

No ano 2000 foi realizado o I Encontro de Coordenadores Estaduais de Alimentação e Nutrição e Centros Colaboradores em Alimentação e Nutrição, no qual foi solicitada a "urgente informatização e uniformização" do SISVAN em nível nacional. Atendendo à demanda, no ano seguinte, a Coordenação-Geral da Política de Alimentação e Nutrição (CGPAN) realizou o diagnóstico da situação da Vigilância Alimentar e Nutricional no país. Foram encontradas diversas limitações, entre elas a baixa cobertura do sistema, a descontinuidade do envio de dados, a falta de integração entre os sistemas existentes e a duplicidade na coleta de informações.

Na tentativa de superar os obstáculos encontrados, iniciou-se em 2002 um processo de reestruturação e informatização da vigilância nutricional sob a direção da CGPAN e do Departamento de Informática do SUS - DATASUS. Nos dois anos seguintes, o processo foi concluído e a Vigilância Nutricional implantada por meio de um sistema informatizado em diversos municípios brasileiros.

Em 2004 foi criado o Programa Bolsa Família, pela Lei ${ }^{\circ}$. 10.836 de 9 de janeiro de 2004, regulamentada pelo Decreto 5.209, de 17 de setembro de 2004 e operacionalizado pela Portaria Interministerial 2.509 de 2004. Este programa prevê também que o SISVAN proverá informações do acompanhamento da saúde das famílias beneficiadas quanto ao acompanhamento do crescimento e desenvolvimento das crianças, conforme o estabelecido no calendário mínimo de consultas para cada faixa etária, e ao cumprimento do calendário vacinal e à realização do pré-natal. Cabe aqui ressaltar que, apesar de as condicionalidades não se restringirem ao acompanhamento do crescimento das crianças, o SISVAN foi o sistema adotado para o acompanhamento das condicionalidades, porque naquele momento foi o único sistema de informações da atenção básica que previa a possibilidade do acompanhamento individualizado.

Em 2006 foi realizado um processo de reavaliação e novo planejamento das ações de Vigilância Alimentar e Nutricional, mediante a realização de uma série de oficinas focadas na caracterização das necessidades de informação para a execução de tais ações na sua plenitude. As oficinas contaram com a participação de membros da CGPAN e foi concebida e facilitada por um analista de planejamento e gestão da FIOCRUZ/Brasília, que também é autor desta publicação.

As oficinas de trabalho resultaram no reconhecimento das limitações, dificuldades e também potencialidades das ações realizadas de Vigilância Alimentar e Nutricional nas três esferas de governo frente aos objetivos idealizados para essa Vigilância. Foram apontadas perspectivas concretas de mudança visando a ampliação e, principalmente, qualificação da vigilância alimentar e nutricional, com o objetivo maior de identificar as situações de risco nutricional e alimentar no âmbito do Sistema Único de Saúde, bem como, no nível coletivo, a realização, com a periodicidade devida, do monitoramento e da avaliação do estado nutricional e alimentar da população brasileira.

\section{O Delineamento da Vigilância Alimentar e Nutricional}

O delineamento da Vigilância Alimentar e Nutricional, no âmbito do SUS, deve ser baseado principalmente nos agravos nutri- 
cionais de maior magnitude para a saúde pública no Brasil e seus fatores de risco. Estes são: o déficit estatural em crianças, além do sobrepeso e obesidade em todas as fases da vida com suas morbidades associadas; a anemia ferropriva, a hipovitaminose A e outras carências nutricionais emergentes como a deficiência de vitamina B1; e a alimentação inadequada, com ênfase no alto consumo de açúcares simples, gorduras saturadas e trans, e baixo consumo de frutas, verduras e legumes.

Baseados nesses problemas, foram delineados os objetivos da Vigilância $\mathrm{Nu}$ tricional e Alimentar. Aqui faz-se necessário diferenciar a vigilância no nível individual e coletivo. Em ambas as situações, a atitude de vigilância é primordial. Isto porque o círculo da vigilância envolve necessariamente uma decisão, uma ação imediata voltada à reversão do agravo identificado no processo. A diferença entre a vigilância individual $\mathrm{e}$ coletiva é que, no primeiro caso, a decisão e a ação podem ocorrer praticamente de forma imediata após a identificação do agravo, enquanto que, coletivamente, esta ação pode levar um tempo maior para ser implementada, freqüentemente devido ao tempo necessário para a compilação, análise e interpretação das informações coletadas.

Os objetivos da Vigilância Nutricional e Alimentar devem ser avaliar e monitorar:

- o estado nutricional de diferentes grupos populacionais;

- as morbidades associadas aos principais desvios nutricionais;

- as carências nutricionais específicas, como aquelas relacionadas à vitamina A, ao ferro, ao iodo e à tiamina;

- a prática de aleitamento materno e a introdução de alimentos complementares;

- a qualidade da alimentação quanto ao consumo de energia, de macro e micronutrientes (principalmente de vitamina A, ferro e iodo), e de grupos alimentares, como frutas, verduras e legumes, gorduras, com destaque para as do tipo trans e saturadas, sódio e açúcares livres.
Nessa perspectiva, a Vigilância Alimentar e Nutricional só pode ser entendida e implementada por uma combinação de estratégias de vigilância epidemiológica. Atualmente as estratégias que a Coordenação-Geral da Política de Alimentação e Nutrição adota para a implementação da vigilância alimentar e nutricional são:

- Sistema Informatizado de Vigilância Alimentar e Nutricional;

- Inquéritos Populacionais Periódicos;

- Análise e cruzamento de informações coletadas por outros Sistemas de Informação da Saúde;

- Realização de Chamadas Nutricionais;

- Acesso à produção científica nacional $\mathrm{e}$ internacional e financiamento de pesquisas.

Cada uma dessas estratégias que compõem a Vigilância Alimentar e Nutricional apresenta potencialidades e limitações que são apresentadas a seguir.

Entende-se que os indicadores oriundos deste rol de informações oferecem o suporte necessário para a análise sistemática das ações e políticas relacionadas à situação alimentar e nutricional da população brasileira, não somente no nível federal, mas também nas esferas estaduais e municipais.

\section{As Estratégias Epidemiológicas que compõem a Vigilância Alimentar e Nutricional}

\section{Sistema Informatizado de Vigilância Alimentar e Nutricional:}

O Sistema Informatizado corresponde a um sistema de informações alimentado no nível local, que tem como objetivo principal promover informação contínua sobre o estado nutricional e alimentar da população vigiada. No momento atual, a população vigiada se refere à população atendida pela Atenção Básica do Sistema Único de Saúde, incluindo os beneficiários do programa de transferência de renda do Governo Federal, o Programa Bolsa Família.

Nesse sentido, atualmente há dois sistemas informatizados, ambos acessados pelas 
Secretarias de Saúde via Internet: o SISVAN Web e o Bolsa Família na Saúde. O primeiro, lançado em dezembro de 2007, apresenta como principais vantagens em relação ao sistema anterior, conhecido como "módulo municipal”, a incorporação das curvas de crescimento da Organização Mundial da Saúde de 2006 e 2007 para a avaliação do estado nutricional de crianças e adolescentes, a possibilidade de registro de marcadores do consumo alimentar nas diferentes idades, além de apresentar uma interface mais fácil para o usuário do sistema.

Os dados de consumo alimentar registrados no sistema são provenientes do preenchimento de determinadas questões, em formulários próprios do SISVAN Web, diferenciadas segundo a fase da vida. Para crianças menores de 6 meses, as questões têm como objetivo caracterizar a prática de aleitamento materno e identificar o tipo de alimentação que a criança está recebendo. Para crianças entre 6 meses e menos de 2 anos, o foco da avaliação de consumo é caracterizar a introdução de alimentos, que deve ocorrer a partir dos 6 meses de idade, e a adoção de comportamento de risco tanto para a ocorrência de anemia como para o desenvolvimento de excesso de peso. As questões para crianças entre 2 anos e menos de 5 anos visam avaliar as práticas alimentares de crianças que já devem ter adotado a alimentação da família. Já no caso de crianças a partir dos 5 anos e para as demais fases da vida, os marcadores de consumo alimentar têm como objetivo identificar com que frequência o entrevistado consumiu alguns alimentos ou bebidas nos últimos 7 dias, que estão relacionados tanto a uma alimentação saudável (exemplo: consumo diário de feijão, frutas, verduras) como a práticas pouco recomendadas (exemplo: consumo freqüente de alimentos fritos e guloseimas).

O SISVAN Web tem apresentado resultados muito satisfatórios, alcançando mais de 9 milhões de acompanhamentos em aproximadamente um ano e meio de utilização. Avaliando-se os dados do ano de 2008, verifica-se uma preponderância de registros do estado nutricional de adultos e crianças (Tabela 1), fase considerada como prioritária por sua vulnerabilidade etária. Em relação aos dados de consumo alimentar, no mesmo período, foram obtidos aproximadamente 31 mil registros de menores de 5 anos de idade e 94 mil registros de indivíduos com 5 anos ou mais, abrangendo as demais fases da vida.

Todas as informações registradas no sistema informatizado, referentes ao estado nutricional e consumo alimentar, são apresentadas em relatórios, no formato de tabelas. Estes servem de subsídio para a realização de ações voltadas ao restabelecimento das condições adequadas de saúde e nutrição da população acompanhada. Atualmente, os relatórios podem ser desagregados até os Estabelecimentos de Saúde dos municípios, incluindo o acompanhamento do histórico individual. Tais dados são importantes e valiosos porque permitem traçar o perfil epidemiológico e nutricional da população e refletem o impacto das ações realizadas em determinadas comunidades.

O segundo sistema informatizado, o Bolsa Família na Saúde, é utilizado para o acompanhamento do registro das condicionalidades da saúde do Programa Bolsa Família, avaliando o acesso desse público às ações básicas de saúde. Entre crianças menores de sete anos, as condicionalidades do setor saúde correspondem ao acompanhamento do crescimento e cumprimento do calendário de vacinação; entre gestantes, é exigida a participação nas consultas do pré-natal e pós-parto. A partir do segundo semestre de 2006, a possibilidade de registrar o estado nutricional dos beneficiários do Programa Bolsa Família com perfil saúde (crianças menores de 7 anos e mulheres em idade fértil) ampliou de forma expressiva o acesso aos dados da vigilância nutricional, sendo que no segundo semestre de 2008, foram recebidos mais de 7,3 milhões de registros de estado nutricional dos beneficiários em todo o Brasil.

Destacam-se algumas potencialidades do Sistema Informatizado, como o nível de desagregação da informação, a possibilida- 
Tabela 1 - Distribuição do número de indivíduos acompanhados com registro no SISVAN Web, segundo fase da vida e Unidade Federativa. Brasil, 2008.

Table 1 - Distribution of the number of individuals followed with record in SISVAN Web, according to the phase of life and State. Brazil, 2008.

\begin{tabular}{|c|c|c|c|c|c|c|}
\hline \multirow[t]{2}{*}{ Unidade Federativa } & \multicolumn{6}{|c|}{ Fase da vida } \\
\hline & Criança & Adolescente & Adulto & Idoso & Gestante & TOTAL \\
\hline Acre & 16.901 & 8.740 & 20.068 & 12 & 369 & 46.090 \\
\hline Alagoas & 99.226 & 50.047 & 102.534 & 228 & 2.292 & 254.327 \\
\hline Amazonas & 85.463 & 39.169 & 73.980 & 207 & 2.107 & 200.926 \\
\hline Amapá & 6.940 & 2.239 & 4.539 & 10 & 160 & 13.888 \\
\hline Bahia & 429.291 & 226.613 & 466.509 & 4.312 & 14.508 & 1.141 .233 \\
\hline Ceará & 274.629 & 197.347 & 406.166 & 1.638 & 6.985 & 886.765 \\
\hline Distrito Federal & 2.476 & 2.283 & 3.878 & 130 & 211 & 8.978 \\
\hline Espírito Santo & 89.453 & 34.332 & 78.746 & 1.724 & 6.021 & 210.276 \\
\hline Goiás & 93.061 & 43.216 & 101.195 & 2.017 & 1.951 & 241.440 \\
\hline Maranhão & 217.173 & 147.282 & 269.635 & 1.502 & 3.728 & 639.320 \\
\hline Minas Gerais & 326.094 & 200.417 & 414.765 & 15.426 & 9.517 & 966.219 \\
\hline Mato Grosso do Sul & 40.598 & 17.293 & 38.388 & 1.690 & 2.435 & 100.404 \\
\hline Mato Grosso & 40.470 & 27.019 & 51.981 & 2.516 & 2.267 & 124.253 \\
\hline Pará & 186.661 & 124.243 & 215.486 & 2.283 & 7.257 & 535.930 \\
\hline Paraíba & 174.252 & 66.705 & 148.600 & 1.387 & 7.587 & 398.531 \\
\hline Pernambuco & 169.520 & 69.790 & 151.438 & 1.161 & 5.382 & 397.291 \\
\hline Piauí & 98.318 & 61.063 & 132.842 & 191 & 2.327 & 294.741 \\
\hline Paraná & 185.836 & 67.228 & 139.235 & 3.938 & 13.849 & 410.086 \\
\hline Rio de Janeiro & 92.240 & 32.151 & 78.091 & 158 & 1.728 & 204.368 \\
\hline Rio Grande do Norte & 78.630 & 49.430 & 106.729 & 276 & 3.084 & 238.149 \\
\hline Rondônia & 28.680 & 13.143 & 34.014 & 6 & 421 & 76.264 \\
\hline Roraima & 9.922 & 2.918 & 7.268 & 26 & 131 & 20.265 \\
\hline Rio Grande do Sul & 161.408 & 54.588 & 118.554 & 8.245 & 8.865 & 351.660 \\
\hline Santa Catarina & 81.860 & 23.360 & 41.388 & 2.833 & 11.632 & 161.073 \\
\hline Sergipe & 46.604 & 27.277 & 54.654 & 186 & 764 & 129.485 \\
\hline São Paulo & 316.284 & 116.164 & 266.251 & 5.916 & 13.299 & 717.914 \\
\hline Tocantins & 42.446 & 27.268 & 56.318 & 757 & 2.124 & 128.913 \\
\hline TOTAL & 3.394 .436 & 1.731 .325 & 3.583.252 & 58.775 & 131.001 & 8.898.789 \\
\hline
\end{tabular}

de de localização exata e real do agravo e a rapidez com que a informação é coletada, consolidada, analisada e interpretada. Hoje, o SISVAN é um instrumento que permite a avaliação mais precisa do estado nutricional dos usuários do SUS, possibilitando a identificação das Unidades Básicas de Saúde com maiores prevalências de determinados agravos.

Recomenda-se que a periodicidade de alimentação do sistema com dados de crianças e gestantes siga o calendário do acompanhamento do crescimento e desenvolvimento infantil e as consultas do pré-natal. Para outras fases do curso da vida e outras situações fisiológicas, sugere-se que seja realizado, no mínimo, um registro por ano, adaptando esta ação à realidade de cada local. Contudo, verifica-se que não há uma periodicidade regular de envio das informações coletadas pelo município para o DATASUS, sendo que esta também não é cobrada ou definida em relação à população atendida por demanda espontânea. Metade dos municípios brasileiros registra informações no SISVAN Web em 2008 e 95\% enviaram dados antropométricos pelo sistema informatizado do Bolsa Família na 
segunda vigência de 2008. Todas as informações registradas nos sistemas são públicas e estão disponíveis nos sítios do DATASUS e da CGPAN.

\section{Inquéritos Populacionais Periódicos:}

Os inquéritos periódicos são definidos como pesquisas de base populacional, abrangendo uma amostra representativa da população sob vigilância, que viabilizam uma "fotografia", isto é, uma informação pontual da situação de saúde do grupo, incluindo a temática de alimentação e de nutrição. Trata-se de uma estratégia que permite uma avaliação periódica das informações de interesse, mas não possibilita um monitoramento contínuo do panorama de interesse. A principal vantagem dessa estratégia é a formação de linhas de base populacionais para proceder à realização de avaliações periódicas subseqüentes. Por outro lado, as limitações incluem: o alto custo e excessivo trabalho operacional, o que impede a sua realização com uma periodicidade reduzida e também a realização abrangendo amostras com representatividade de estados e municípios, o que impossibilita a localização exata do problema.

O fomento à realização periódica de inquéritos populacionais é uma das estratégias da Vigilância Alimentar e Nutricional conduzidas pelo Ministério da Saúde. Em 2008, foram divulgados dados da Pesquisa Nacional de Demografia e Saúde (PNDS), realizada em 2006. A coleta de dados de outros dois inquéritos populacionais, também financiados pelo Ministério da Saúde, foi iniciada em 2008. Estes correspondem aos módulos de consumo alimentar e de antropometria da Pesquisa de Orçamento Familiar (POF 2008-2009), pesquisa realizada periodicamente pelo Instituto Brasileiro de Geografia e Estatística (IBGE), e a Pesquisa de Avaliação do Impacto da Iodação do Sal (PNAISAL), em execução pelo Centro de Pesquisas Epidemiológicas do Departamento de Medicina Social da Faculdade de Medicina da Universidade Federal de Pelotas.

A realização periódica de estudos po- pulacionais é fundamental porque aponta as tendências dos agravos em nível populacional. A PNDS/2006 apontou dados bastante animadores quanto à redução da desnutrição em menores de cinco anos, na última década no país. A pesquisa revelou que o déficit de peso por idade caiu de 5,7\% para $1,7 \%$. O déficit de altura versus idade teve redução de $13 \%$ para $7 \%$ entre 1996 e 2006. Esta redução é maior ainda, chegando a $70 \%$ no período, quando avaliada apenas a região nordeste.

Atualmente, os únicos dados nacionais disponíveis sobre o consumo direto de alimentos da população datam de 1975 , quando o IBGE realizou o Estudo Nacional de Despesas Familiares (ENDEF). Na última edição da POF, em 2003, o IBGE avaliou medidas de peso e estatura nas diferentes fases da vida dos brasileiros e a disponibilidade de alimentos. A POF 2008-2009 tem como novidade o levantamento do consumo direto dos alimentos, medido pelo registro alimentar da população acima de 10 anos de idade. Do total de 60 mil domicílios pesquisados, $1 / 3$ deles será avaliado quanto ao consumo alimentar individual. A pesquisa permitirá a investigação de um conjunto de informações para subsidiar análises sobre o grau e a qualidade do atendimento à necessidade mais básica do brasileiro sob a ótica do consumo. O Ministério da Saúde e o IBGE detalharão os padrões e hábitos de consumo de alimentos associados a levantamentos de medidas antropométricas nas diversas regiões do país, em diferentes estratos de renda e grupos populacionais.

E, por fim, o último estudo populacional em curso vem a ser a Pesquisa Nacional para Avaliação do Impacto da Iodação do Sal - PNAISAL. Trata-se de um inquérito nacional que tem por objetivo avaliar o impacto da iodação do sal consumido no Brasil, de modo a proporcionar a segurança no processo de fortificação, tanto no que diz respeito à eliminação dos Distúrbios por Deficiência de Iodo (DDI) quanto no que diz respeito à prevenção do surgimento de doenças relacionadas ao consumo excessivo desse micronutriente. 


\section{Análise e cruzamento das bases de dados:}

A análise e o cruzamento periódico das bases de dados nacionais também devem ser considerados como uma estratégia da Vigilância Alimentar e Nutricional. Esta ação pode trazer informações quanto à morbidade e mortalidade associadas à alimentação e nutrição na população brasileira. Trata-se de uma análise anual de diferentes bases de dados nacionais (SINASC, SIM, SIAB, SIA, SIH, Hiperdia, SIS-Pré-Natal), o que pode ser implementado pela CGPAN em parceira com a Secretaria de Vigilância em Saúde (SVS) e o DATASUS.

Esta estratégia de vigilância alimentar e nutricional tem como vantagem o baixo custo, o fato de que a morbidade e a mortalidade são aspectos relacionados à alimentação e nutrição, a possibilidade de avaliação antes e depois de intervenções específicas, além do fato de que as informações podem ser desagregadas até os municípios. Contudo, as principais limitações são a exigência de recursos humanos especializados no tratamento com bancos de dados, a qualidade duvidosa das informações registradas, a especificidade das variáveis, a periodicidade de coleta de alguns sistemas e os vieses de cobertura populacional de outros.

Estas análises permitem investigar a partir de cruzamentos específicos dos agravos nutricionais com sistemas de internação, tanto hospitalar como ambulatorial. Esta ação pode trazer informações quanto à morbidade e à mortalidade associadas à alimentação e nutrição na população brasileira.

\section{Realização de Chamadas Nutricionais:}

As Chamadas Nutricionais são estratégias vinculadas às Campanhas Nacionais de Imunização, que buscam a mobilização para fins de atitude de vigilância das secretarias municipais e estaduais de saúde para a importância do acompanhamento do crescimento de crianças, mas também a realização de levantamento de informações antropométricas e de consumo alimentar em crianças menores de cinco anos.

Faz-se necessário contextualizar a experiência brasileira na realização desta
Estratégia. A iniciativa de realizar a primeira grande Chamada Nutricional em 2005 partiu do Ministério do Desenvolvimento Social e Combate à Fome (MDS) e foi endossada pelo Ministério da Saúde. No ano de 2005, foi realizada a Chamada Nutricional no Semi-Árido e, em 2006, a Chamada Nutricional Quilombolas.

Em 2007, a CGPAN teve a iniciativa para a realização da Chamada Nutricional em todos os Estados da Região Norte. A ação foi realizada em parceria com o Programa Nacional de Imunizações da Secretaria de Vigilância em Saúde do Ministério da Saúde (PNI/SVS), Ministério do Desenvolvimento Social e Combate à Fome, Fundo das Nações Unidas para a Infância (UNICEF) e, principalmente, com as secretarias de saúde dos Estados da Região Norte. Foram avaliados mais de 14 mil menores de 5 anos, através de uma amostra randomizada e representativa da região. Verificou-se que $23,1 \%$ e $5,2 \%$ das crianças avaliadas apresentavam, respectivamente, déficits de estatura e de peso ${ }^{7}$.

As Chamadas Nutricionais apresentam uma lógica parecida com os Inquéritos Populacionais quanto ao cuidado metodológico; no entanto, essa estratégia, apresenta algumas diferenças como a possibilidade de levantar informações com desagregação estadual - o que dificilmente pode ser realizado em um inquérito populacional devido ao custo. Outra característica é a possibilidade de que as informações coletadas sejam rapidamente analisadas e divulgadas. Mas a mais importante qualidade dessa estratégia é a sensibilização e mobilização da população e dos gestores da saúde sobre a importância do acompanhamento do crescimento e desenvolvimento das crianças.

\section{Acesso à produção científica e}

financiamento de pesquisas:

$\mathrm{O}$ acesso à produção científica corresponde a um levantamento periódico, sistemático e exaustivo da produção científica nacional sobre a situação alimentar e nutricional da população brasileira e/ou do desenvolvimento dos programas sociais. Como estratégia de vigilância alimentar e 
nutricional, devem ser acessados estudos nacionais e internacionais que apóiem ou complementem com dados epidemiológicos o monitoramento da população.

Considerando a importância desse acesso para o direcionamento das ações de alimentação e nutrição, a CGPAN tem realizado e incentivado o acesso à produção científica nacional e internacional para os diversos segmentos populacionais, em especial aos trabalhadores do SUS ligados ao tema, formadores da educação profissional e comunidade científica. Utiliza diversos meios de disponibilização objetivando o maior alcance das informações (publicações produzidas pelo SUS, revistas científicas, bibliotecas virtuais e sites governamentais, entre outros).

Adicionalmente, a CGPAN tem incentivado e apoiado a realização de estudos e pesquisas que permitam conhecer aspectos gerais e específicos da situação alimentar e nutricional do país, avaliar a contribuição dos fatores causais envolvidos e indicar medidas mais apropriadas para prevenção e controle dos problemas de saúde e nutrição.

Entre as diversas instituições de ensino e pesquisa apoiadas, destacam-se os Centros Colaboradores e de Referência em Alimentação e Nutrição e instituições governamentais mediante a realização de Editais de Pesquisas. Nessa configuração de fomento aos estudos e pesquisas, a CGPAN conta com a parceria do Departamento de Ciências e Tecnologia (MS) e também do Conselho Nacional de Desenvolvimento Científico e Tecnológico (CNPq).

Até então foram conduzidos dois editais de pesquisas. Em 2004 foi lançado o CT-SAÚDE/MCT/CNPq/MS/DECIT n ${ }^{\circ}$. 030/2004. Para esse edital concorreram 461 projetos, sendo que 85 foram financiados, $\mathrm{o}$ que significou um total de $\mathrm{R} \$ 4.337 .162,22$. Em 2005 a CGPAN publicou o Edital MCT/ CNPq/MS-SCTIE-DECIT/SAS-DAB n ${ }^{\circ}$. 051/2005 - "Alimentação, Nutrição e Promoção da Alimentação e Modos de Vida Saudáveis", com um desenho de investigação mais restrito, sendo voltado para a promoção da alimentação e modos de vida saudáveis. A demanda bruta de projetos foi de 300, sendo 97 financiados, o que significa um investimento vertical de $\mathrm{R} \$ 4.943 .444,38$.

\section{Publicização de informações da Vigilância Alimentar e Nutricional}

Diante da realização de uma série de pesquisas e da compilação dos dados mencionados fez-se necessária a idealização de um informativo que agregasse informações de saúde e nutrição do País com o objetivo de facilitar a capacidade de formulação, gestão e avaliação de políticas e ações públicas dirigidas à melhoria da qualidade de vida e saúde da população. O Boletim SISVAN é um informativo eletrônico, atualizado quadrimestralmente, com o propósito de promover a disponibilidade adequada e oportuna de informações para a gestão. Este boletim está disponível no sítio eletrônico da CGPAN (www.saude.gov.br/nutricao).

Paralelamente, no início de 2008, a CGPAN propôs à REDE RIPSA a criação de um Comitê Temático Interdisciplinar de Indicadores de Alimentação e Nutrição com vistas a proporcionar maior visibilidade às informações de nutrição e saúde que estão sendo produzidas nacionalmente, pelos inquéritos populacionais e sistemas de informação. A RIPSA é a Rede Interagencial de Informações para a Saúde, responsável pela articulação de entidades representativas dos segmentos técnicos e científicos nacionais envolvidos na produção e análise de dados, para viabilizar parcerias que propiciem informações úteis ao conhecimento e à compreensão da realidade sanitária brasileira e suas tendências.

A Rede atua com base em Acordo de Cooperação firmado entre o Ministério da Saúde e a Organização Pan-Americana da Saúde (OPAS), balizada por um conjunto de indicadores e dados básicos, periodicamente atualizados e aperfeiçoados, sobre aspectos demográficos, socioeconômicos, de mortalidade, de morbidade e fatores de risco, de recursos e de cobertura de ações e serviços. Integram a RIPSA cerca de 21 instituições nacionais representativas dos seg- 
mentos de produção de informações estrito senso, de gestão do sistema de saúde e de desenvolvimento científico e tecnológico.

O Comitê Temático Interdisciplinar de Indicadores de Alimentação e Nutrição tem como objetivos definir, organizar, analisar e propor um rol de indicadores de Alimentação e Nutrição para as diversas fases do curso da vida, em vários níveis de agregação geográfica, que sejam capazes de orientar as políticas públicas inclusivas e que levem à redução das desigualdades.

\section{Desafios para a implementação das ações do SISVAN}

Desde a idealização do sistema de vigilância alimentar e nutricional até hoje, muitas mudanças e avanços ocorreram. $\mathrm{O}$ sistema atualmente possui importantes ferramentas que, se utilizadas corretamente, possibilitam inúmeras inferências relativas não só ao estado nutricional como ao consumo alimentar da população brasileira. A informatização do sistema possibilitou agilidade na consolidação das informações em diferentes níveis e áreas de abrangências. A inserção dos dados nos municípios, de forma constante e em volume crescente, reflete na alimentação contínua no banco de dados centralizado nacionalmente.
Atualmente, as capacitações do SISVAN visam sua implementação e ampliação, bem como a expansão do uso do sistema informatizado, em todos os municípios brasileiros. O próximo passo é a capacitação para a coleta de dados e registro das informações com maior qualidade, além de melhorar o conhecimento dos profissionais envolvidos na interpretação dos dados da vigilância alimentar e nutricional de sua população. Essas medidas aumentarão a confiabilidade da base de dados gerada pelo SISVAN e farão com que o sistema se torne um referencial na avaliação do perfil alimentar e nutricional da população brasileira, que é o maior desafio enfrentado atualmente.

Outro desafio para o SISVAN é a consolidação e o uso das informações disponibilizadas para a gestão de ações e recursos voltados a ações de alimentação e nutrição e de estratégias gerais de saúde. Os gestores e profissionais ligados à área da saúde precisam se apoderar dos dados do sistema. Todos os profissionais envolvidos desde a coleta de dados, inserção de dados no sistema, gerenciamento do banco de dados e dos relatórios formam uma rede responsável pela geração dos dados e devem trabalhar em consonância e focados no objetivo comum da manutenção do sistema.

\section{Referências}

1. Brasil. Ministério da Saúde. Secretaria de Atenção à Saúde. Departamento de Atenção Básica. Política Nacional de Alimentação e Nutrição (2a ed. rev.) Brasília; 2008.

2. Santana LAA, Santos SMC. Sistema de Vigilância Alimentar e Nutricional na implementação do programa Leite é Saúde: avaliação em municípios baianos. Rev Nutr 2004; 17(3): 282-90.

3. Vasconcelos FAG. Combate à fome no Brasil: uma análise histórica de Vargas a Lula. Rev Nutr 2005; 18(4): 439-57.

4. Batista Filho M, Lucena MAF, Evangelista MLM. A vigilância alimentar e nutricional no Brasil. Cad Saúde Pública 1986; 2(3): 349-58.
5. Arruda BKG, Arruda IKG. Marcos referenciais da trajetória das políticas de alimentação e nutrição no Brasil. Rev Bras Saúde Matern Infant 2007; 7(3): 319-26.

6. Brasil. Fundação IBGE (Instituto Brasileiro de Geografia e Estatística). Estudo Nacional de Despesa Familiar ENDEF. Rio de Janeiro: IBGE; 1976.

7. Brasil. Ministério da Saúde. Secretaria de Atenção à Saúde. Departamento de Atenção Básica. CoordenaçãoGeral da Política de Alimentação e Nutrição. Chamada Nutricional da Região Norte - 2007: Resumo Executivo Disponível em http://nutricao.saude.gov.br/ documentos/resumo_chamada_nutricional_regiao_ norte.pdf [Acessado em 22 de maio de 2009].

Recebido em: 01/09/09 Aprovado em: 05/10/09 